Quaderni di Geografia Cahiers de Gécongaty rataphie

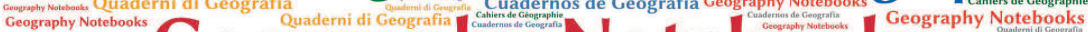
Cuadernos de Geografia Geography Notebooks $U 201000$ Cahiers de Géographi Cahiers de Géographie cahiers de Géographie Cuadernos de Geografía Cahiers de Géographie Cahiers de Géographie Cuadernos de Geografía Geography Notebooks

\author{
$4(2021)$ \\ 1 \\ Teatro di suoni. \\ Spazi acustici teatrali e territoriali
}

A cura di

Martino Mocchi, Lorena Rocca, Demis Quadri and Carlotta Sillano

EDITORIAL

Teatro di suoni per l'attaccamento ai luoghi. Uno sguardo geografico 11

Lorena Rocca

Per un teatro di suoni. Riflessioni su possibili dimensioni sonore nelle 23 creazioni site-specific di physical theatre

Demis Quadri

INTRODUCTION

Teatro di suoni. Spazi acustici teatrali e territoriali

Demis Quadri e Lorena Rocca

SPECIAL Issue

I suoni di Mantova come strumenti di interpretazione del paesaggio.

Tra turismo sostenibile ed educazione al patrimonio culturale

Valeria Pecorelli, Franca Zuccoli, Alessandra De Nicola, Enrico Squarcina

Il paesaggio sonoro campano tra contemporaneità e nuove forme

di progettualità turistica

Germana Citarella 
La narrazione spettacolarizzata del paesaggio sonoro.

Da Giuseppe Chiari a Philip K. Dick e oltre

Francesco Michi

Musica di paesaggi sonori. Enunciazione, risignificazione, comunicazione

Carlotta Sillano

Camminare per ascoltare. Partiture invisibili del territorio abitato

Elisabetta Senesi

Il paesaggio sonoro in relazione. Suono, movimento e immagini per stimolare complessità percettiva Angela Calia

Groove Fields. Understanding the Dance Floor from an Art-Based Research Perspective

Sebastian Mattbias

Il silenzio come esperienza trasformativa. L'importanza del silenzio nella meditazione e in ambito professionale

Sebastiano Caroni

Progettare il silenzio. Una lettura acustica dell'ex villaggio sanatoriale 125 Morelli a Sondalo

Martino Mocchi

Voicing One's Will. Theatre as Audio-Visual Hypotyposis of the Poetic

Michael Groneberg

Music and Clowning in Europe, 20th-21st centuries

Anna Stoll Knecht

Il paesaggio sonoro nella composizione musicale. Un percorso bibliografico

Stefano Alessandretti

\#exploreART: il labirinto di A. Pomodoro e i bambini. Un progetto di fruizione condivisa con percorsi sensoriali partecipati Alessandra De Nicola, Franca Zuccoli 


\section{OTHER EXPLORATIONS}

Il rumore lontano. Intervista a Lorena Rocca

a cura di Martino Mocchi

Re Cervo. Intervista a Antonella Astolfi

a cura di Krizia Bonaudo e Demis Quadri

Centovalli-Centoricordi. Intervista a Oliviero Giovannoni a cura di Krizia Bonaudo e Demis Quadri

Alla ricerca di un metodo: Open Space Technology 



\title{
Il paesaggio sonoro nella composizione musicale. Un percorso bibliografico
}

\author{
Stefano Alessandretti \\ Compositore e performer \\ DOI: https://doi.org/10.7358/gn-2021-001-ales
}

\begin{abstract}
Over the last few decades there has been a proliferation of different fields of study directly derived from what can now be defined as a real discipline: the soundscape. Because of the growing number of new subjects, it becomes really challenging to get out of the multitude of publications, authors and terminologies, in order to create a synthetic and exhaustive bibliography dealing with the primary subject from which the various fields of study branched out. Starting from this difficulty this work was born, a bibliographic research aiming to direct the reader towards a targeted literature in the field of soundscape and music composition.
\end{abstract}

Keywords: soundscape annotated bibliography; soundscape primary sources; soundscape composition; soundscape overview.

Parole chiave: bibliografia ragionata paesaggio sonoro; fonti principali paesaggio sonoro; composizione e paesaggio sonoro; inquadramento paesaggio sonoro.

\section{LA RICERCA E I SUOI CRITERI}

Negli ultimi decenni si è assistito alla proliferazione di numerosi campi di studio direttamente derivati da quella che è ormai possibile definire come una vera e propria disciplina: il paesaggio sonoro o soundscape. Sebbene il termine sia stato formalizzato in tempi relativamente recenti 
(Schafer 1977) ${ }^{1}$, è possibile rintracciare numerose riflessioni ante litteram che testimoniano un primigenio interesse per le sonorità dei paesaggi già a partire dall'inizio dello scorso secolo. Basti pensare alle esperienze artistiche del futurismo, al grande cambiamento segnato dal passaggio dal cinema muto a quello sonoro o alle importanti sperimentazioni della musica concreta; tutti episodi che appartengono alla prima metà del secolo scorso.

Come conseguenza della diffusione di queste nuove materie di studio, risulta sempre più impegnativo districarsi tra la moltitudine crescente di pubblicazioni, autori e nuove terminologie nel campo - spesso necessariamente ambigue - con lo scopo di creare un percorso bibliografico sintetico ed esaustivo, che tratti della materia di studio da cui le varie branche si sono ramificate: il paesaggio sonoro in relazione alla composizione musicale.

A partire proprio da questa difficoltà nasce il presente lavoro di ricerca bibliografica, che ha il fine di indirizzare lo studioso, il ricercatore o il semplice appassionato verso una mirata letteratura nel campo.

Per favorire ciascun ambito di interesse, sia esso storico, estetico-filosofico, sociologico-culturale, tecnico o musicale, i principali ${ }^{2}$ risultati della ricerca sono stati organizzati in 5 capitoli, coincidenti con le seguenti categorie di studio:

- inquadramento estetico-filosofico; vi sono raggruppati quei testi che ripercorrono le diverse riflessioni estetiche e filosofiche sull'ascolto e la natura della musica;

- inquadramento storico e socio-culturale; quella letteratura che permette di comprendere l'evoluzione storica, sociale e culturale nel rapporto tra l'uomo e l'arte dei suoni;

- il paesaggio sonoro; una raccolta di testi che tratta specificatamente del paesaggio sonoro e delle nuove modalità di ascolto musicale.

- composizione del paesaggio sonoro; un insieme di opere focalizzate sulla composizione elettroacustica e i suoi linguaggi;

- registrazione sul campo (field recording) bibliografia consigliata nell'ambito dell'arte della registrazione sul campo, indispensabile pratica nella composizione di qualsiasi paesaggio sonoro.

Inoltre, come appendice al lavoro, è stato incluso un elenco di articoli

${ }^{1} \grave{E}$ possibile rintracciare fonti e autori che utilizzavano il termine soundscape prima di Schafer: lui stesso attribuisce il termine all'urbanista Michael Southworth (1967).

2 Tutti i testi esclusi dalla trattazione ma che possono fungerne da complemento, sono comunque inseriti nella bibliografia. 
scientifici, validi come approfondimenti nelle specificità degli ambiti di studio affrontati.

Nella stesura della ricerca si è cercato, ove possibile, di includere il maggior numero di testi in lingua italiana, mentre la ricerca delle fonti ha seguito tre principali criteri metodologici, così elencati in ordine di incidenza:

- fonti bibliografiche nidificate; partendo dalle pubblicazioni di riconosciuta importanza, sono state consultate le fonti citate, in queste le ulteriori fonti a cui si rimandava e via dicendo, fino a costituire una sorta di classifica delle pubblicazioni in funzione del numero di citazioni accumulate nella ricerca;

- fonti bibliografiche indicizzate nell'archivio Opac; consultazione del catalogo informatizzato delle biblioteche (On-line Public Access Catalogue);

- ricerche tematiche sul web; consultazione dei principali motori di ricerca utilizzando differenti parole chiave (per tematica, autore o istituzione).

\section{INQUADRAMENTO ESTETICO-FILOSOFICO}

«Da venticinque secoli la cultura occidentale cerca di guardare il mondo. Non ha capito che il mondo non si guarda, si ode. Non si legge, si ascolta» (Attali 1978, 7); con queste parole il noto saggista francese Jacques Attali apriva il suo libro Rumori. Saggio sull'economia politica della musica, pubblicato in Francia nel 1977 e tradotto in italiano l'anno successivo. Con questo testo che sembra quasi finito nel dimenticatoio, sebbene sia stato precursore di molti dei temi sviluppati da illustri personalità negli anni a seguire, Jacques Attali reclamava il valore profetico della musica e si prefiggeva il compito di sottolinearne i suoi aspetti economici e politici ${ }^{3}$. A partire dal movimento marxista e da quello post-strutturalista, le riflessioni dell'autore si concentrano sul ventennio precedente gli anni Settanta, intrecciando un'accurata analisi su argomenti quali le trasformazioni della produzione, le pratiche musicali, il ruolo del segno, il rumore e l'egoismo dell'atto musicale, creando fondamentali rimandi alle opere di autori come Michel Foucault, Jean Baudrillard, Ferruccio Rossi-Landi o René Girard.

${ }^{3}$ L'accento è posto sulla rivoluzione tecnologica che l'autore è stato in grado di recepire solamente attraverso l'analisi della pratica musicale. 
Di maggior rilievo da un punto di vista filosofico - tanto da suscitare una lunga serie di dibattiti in seguito alla sua pubblicazione - è invece l'opera di Jean-Luc Nancy All'ascolto (Nancy 2004). Si tratta di un testo fondamentale per coloro che intendono investigare le posizioni della filosofia nei confronti dell'ascolto, della nascita e decadenza del suono, dei suoi rimandi, dei suoi luoghi e dei suoi significati; una profonda e problematica valutazione dei fulcri concettuali che sono insiti nelle categorie che l'accadere del suono mette in atto. Nello svolgersi delle argomentazioni il filosofo analizza le sperimentazioni più audaci nel campo artistico (musicale e non), in modo particolare quelle incentrate sull'ausilio tecnologico come la possibilità di scomposizione/ricomposizione del materiale sonoro.

Parallelamente alla pubblicazione di Nancy, vi è un altro testo altamente esaustivo, stavolta incentrato sui fondamenti degli attuali dibattiti filosofici, The Aesthetics of the Natural Environment (Carlson and Berleant 2004), una raccolta di sedici saggi che è in grado di offrire un raffinato esame dell'estetica ambientale contemporanea ${ }^{4}$.

A metà strada tra dissertazione estetico-filosofica e resoconto storico troviamo due pubblicazioni complementari alle precedenti, Listening through the Noise: The Aesthetics of Experimental Electronic Music (Demers 2010) e Electrosound. Storia ed estetica della musica elettroacustica (Fronzi 2013). Entrambe hanno la peculiarità di includere nella trattazione quei generi musicali extra colti che spesso risultano estromessi dalle riflessioni estetiche - se non addirittura dai semplici resoconti storici ${ }^{5}$ - e che per tale motivo possono aiutare il lettore ad avere una visione d'insieme più ampia, che non escluda i sottogeneri e le sottoculture più influenti.

4 Alcuni di questi saggi erano già stati precedentemente pubblicati nel 1998, nel Journal of Aesthetics and Art Criticism. Essendo difficile descrivere la totalità delle argomentazioni per ciascun contributo, mi limiterò a riportare di seguito i titoli e gli autori di ogni saggio incluso nella pubblicazione: Hepburn R., Contemporary Aesthetics and the Neglect of Natural Beauty; Carlson A., Appreciation and the Natural Environment; Berleant A., The Aesthetic of Art and Nature; Carroll N., On Being Moved by Nature: Between Religion and Natural History; Godlovitch S., Icebreakers: Environmentalism and Natural Aesthetics; Saito Y., Appreciating Nature on Its Own Terms; Brady E., Imagination and the Aesthetics Appreciation of Nature; Eaton M., Fact and Fiction in the Aesthetics Appreciation of Nature; Rolston H., The Aesthetics Experience of Forests; Foster C., The Narrative and the Ambient in Environmental Aesthetics; Moore R., Appreciating Natural Beauty as Natural; Fisher J., What the Hills are Alive With: In Defense of the Sounds of Nature; Crawford D., Scenery and the Aesthetics of Nature; Heyd T., Aesthetic Appreciation and the Many Stories about Nature; Sepänmaa Y., Environmental Stories: Speaking and Writing Nature.

${ }^{5} \mathrm{Ci}$ si riferisce a generi musicali come la Disco o la Techno. 


\section{INQUADRAMENTO STORICO E SOCIO-CULTURALE}

Per poter comprendere al meglio i fattori che hanno portato alle profonde riflessioni sull'ascolto appena citate, è necessario rivolgere l'attenzione su altri importanti aspetti di natura sociologica, storica, antropologica e musicologica; sono gli argomenti discussi in Paesaggi sonori. Musica, voci, rumori: l'universo dell'ascolto (Bull and Back 2008). Si tratta di una raccolta di contributi di vari autori, di carattere multidisciplinare, in cui è possibile tracciare paralleli tra le trasformazioni sociali e la fruizione musicale, capire le interazioni tra cultura, tempo e musica o i legami tra coesione comunitaria e pratica musicale, giungendo fino alla tirannia della visione e la presa di coscienza del suono come strumento di indagine.

Una panoramica simile ma più esaustiva riguardo l'influenza tra le diverse arti nel XX secolo è offerta da Noise, water, meat: a bistory of sound in the arts (Kahn 1999). Il testo affronta le situazioni storiche, sociali e politiche che hanno dato vita alle riflessioni artistiche sul rumore nel campo del cinema, della pittura, della letteratura e della musica, con attenzione all'opera di autori come: Dziga Vertov, Sergei Eisenstein, Grigori Alexandrov, James Joyce, William Burroughs, Gerhard Richter, John Cage e Pierre Schaeffer. Una interessante prospettiva che guarda al rumore come valida via di fuga dalle rigide regole della comunicazione e della narrazione, estrapolandolo dalla consueta fenomenologia musicale.

Di carattere prettamente musicale è l'antologia The book of Music and Nature: an anthology of Sounds, Words, Thoughts (Rothenberg and Ulvaeus 2009) che si basa sul tema della musica e della natura, raccogliendo i contributi di artisti, maestri e pensatori della nostra epoca, come: John Cage, Hazrat Inrayat Khan, Pierre Schaeffer, Rainer Maria Rilke, Toru Takemitsu, Brian Eno, Pauline Oliveros, David Toop, Hildegard Westerkamp, Evan Eisenberg, Rafi Zabor, Alejo Carpentier e Junichiro Tanazaki; una collezione che comprende saggi, illustrazioni, suoni e musiche ${ }^{6}$, intesa a celebrare il nostro rapporto con i paesaggi sonori naturali.

\section{Il PAESAGgio SONORO}

La presenza di un nutrito numero di pubblicazioni in questo capitolo nodale della ricerca bibliografica suggerisce la presentazione delle fonti per

${ }^{6} \grave{E}$ disponibile una serie di materiali sonori online, all'indirizzo http://www.wesleyan.edu/wespress/musicandnaturecd/. 
autore, piuttosto che per titoli o ambiti di studio specifici. La figura più importante è senza dubbio quella di Raymond Murray Schafer, vero e proprio fondatore della disciplina in esame; le opere minime a cui riferirsi sono: The Music of the Environment (Schafer 1973), Il paesaggio sonoro (Schafer 1985) e La nuova orchestra: l'universo sonoro (Schafer 1988). Gli argomenti contenuti nei tre testi hanno sensibilizzato il mondo intero all'ascolto del suono ambientale e rappresentano la nascita dell'ecologia acustica e del concetto di paesaggio sonoro.

«Only a total appreciation of the acoustic environment can give us the resources for improving the orchestration of the world. Ear cleaning in the schools to eliminate audiometry in factories. Clairaudience, not ear muffs» (Schafer 1973, 4). E in seguito: «ecco la nuova orchestra: l'universo sonoro! Ed ecco i suoi nuovi musicisti: chiunque e qualsiasi cosa sappiano emettere un suono» (Schafer 1985, 16).

La fondazione del World Soundscape Project presso la Simon Fraser University, ad opera dello stesso Schafer, ha permesso la costituzione di un eccellente gruppo di studenti e compositori che hanno affiancato e proseguito le ricerche dell'autore; tra le diverse personalità spicca il nome di Hildegard Westerkamp, autrice di Listening and Soundmaking: a Study of Music as Environment (Westerkamp 1988) ${ }^{7}$ - e Soundscape Composition: Linking Inner and Outer Worlds (Westerkamp 1999), entrambi disponibili online all'interno del sito della Simon Fraser University. Nel primo titolo, in cui la trattazione si muove intorno al lavoro di Attali, Adorno, Barthes, Deleuze e Kristeva, viene sostanzialmente sostenuta l'importanza dell'equilibrio tra ascolto e produzione musicale per la salute della psiche umana, nonché l'essenzialità del processo creativo nella lotta contro il sovraccarico acustico dovuto al cambiamento dei paesaggi sonori urbani ${ }^{8}$.

I have seen creativity at a way of restoring confidence in our listening and soundmaking capacity and argued that the immediacy of childhood existence (where it can still be witnessed) can give us cues for an actively creative existence as well as a physical relationship to the world. (Westerkamp 1988, 147)

Nel secondo titolo, invece, le stesse tematiche sono trattate da un punto

\footnotetext{
${ }^{7}$ Si tratta della tesi di laurea dell'autrice, Master of Arts (Communication).

${ }^{8}$ L'autrice parla di input e output riferendosi rispettivamente all'ascolto e la produzione sonora.
} 
di vista compositivo, concentrando le argomentazioni sul rapporto tra l'intenzione dell'autore e la percezione dell'ascoltatore.

A soundscape composition is always rooted in themes of the sound environment. It is never abstract. Recorded environmental sounds are its 'instruments', and they may be heard both unprocessed and processed. Some works are created entirely with unprocessed sounds and their compositional process occurs in the specific ways in which the sounds are selected, edited, mixed and organized. (Westerkamp 1999)

Un'altra autrice che ha fondato la propria ricerca nel campo della percezione uditiva è Pauline Oliveros che con il suo Deep Listening: A Composer's Sound Practice (Oliveros 2005) fornisce delle interessanti intuizioni sul modo in cui la coscienza possa essere influenzata dalla profonda attenzione verso l'ambiente sonoro, pratica che definisce per l'appunto deep listening e che si è adoperata a diffondere attraverso laboratori, seminari e ritiri di ascolto in giro per il mondo. «Deep listening for me is learning to expand the perception of sounds to include the whole space/time continuum of sound encountering the vastness and complexities as much as possible» (Oliveros 2005, 23).

Tra le ulteriori fonti è possibile rintracciare Musica e suoni dell'ambiente (Mayr 2001), Ecologia della musica (Colimberti 2004), Il paesaggio e il silenzio (Turri 2004), Il paesaggio come teatro. Dal territorio vissuto al territorio rappresentato (Turri 2006) e Ways of listening: An Ecological Approach to the Perception of Musical Meaning (Clarke 2008), segnalo l'antologia Hearing places: sound, place, time and culture (Bandt et al. 2007), utile a integrare e ampliare le riflessioni degli autori sopra menzionati. L'opera, che riunisce i contributi di 37 studiosi e artisti che si interrogano sul concetto di 'luogo musicale', sul significato dei suoni e su quello dell'ascolto, include un cd con 36 tracce audio e ben 34 saggi.

\section{Composizione del PAESAggio SONORO}

Sebbene siano numerose le fonti letterarie nel campo del paesaggio sonoro, soltanto una piccola percentuale di esse tratta dell'estetica musicale elettroacustica da un punto di vista analitico e organizzativo. I testi di seguito raccolti saranno perciò un'utile risorsa per chi si interessa dell'arte di organizzare i suoni elettroacustici perché focalizzati su argomenti musicali come il materiale, la struttura, il linguaggio e la forma. 
The Language of Electroacoustic Music (Emmerson 1986) è uno dei primi testi scritti in materia di composizione elettroacustica ed è organizzato in tre sezioni principali: Culture and Language, Materials and Language e The Influence of New Technology. L'edizione contiene diversi saggi ad opera dei più importanti compositori contemporanei ${ }^{9}$.

Di pochi anni più recente è invece Audible Design: A Plain and Easy Introduction to Sound Composition (Wishart 1994) che si basa su tre assunti basilari: qualsiasi tipo di suono può essere il materiale di partenza per una composizione musicale; le modalità secondo le quali tale suono può essere trasformato sono limitate esclusivamente dall'immaginazione del compositore; la struttura musicale dipende dalle relazioni percepibili tra i materiali sonori. La trattazione prevede quindi la discussione generale su diverse tipologie di materiali sonori e le trasformazioni risultanti dal loro trattamento per mezzo di processi ben definiti ${ }^{10}$.

Già pubblicato nel 1984 e in seguito riveduto, ampliato e accompagnato da un cd-rom nell'edizione successiva, è Acoustic Communication (Truax 2001) che tratta della comunicazione acustica in un quadro multidisciplinare ed è completato da una versione digitale del manuale di ecologia acustica (Handbook for acoustic ecology), un'enciclopedia ipertestuale su quasi tutto ciò che ha a che fare con il suono (percezione uditiva, acusmatica, psicoacustica ecc.) con numerosi esempi sonori e grafici.

Vi sono infine due ulteriori letture che per chiarezza e accessibilità dei contenuti possono essere utili anche ai fini pedagogici, Understanding the Art of Sound Organization (Landy 2007) e Making Music with Sounds (Landy 2012); entrambi i testi offrono un programma di apprendimento che, a partire dalla consapevolezza uditiva, attraversa la scoperta e l'organizzazione dei suoni giungendo fino ai mezzi di generazione e manipolazione e sono supportati dall'esaustiva risorsa online EARS - ElectroAcoustic Resource Site, a opera dello stesso autore.

${ }^{9}$ I contributi sono così organizzati: Emmerson S., Introduction; The Relation of Language to Materials; Boulez P., Technology and the Composer; Wishart T., Sound Symbols and Landscapes; Smalley D., Spectro-morphology and Structuring Processes; Keane D., At the Threshold of an Aesthetic; Pennycook B., Language and Resources: A New Paradox; McNabb M., Computer Music: Some Aesthetic Considerations; Truax B., Computer Music Language Design and the Composing Process; Harvey J., The Mirror of Ambiguity; Machover T., A Stubborn Search for Artistic Unity.

${ }^{10}$ In relazione a ciò, i capitoli sono così organizzati: Introduction, The Nature of Sound, Pitch, Spectrum, Onset, Continuation, Grain, Streams, Sequences, Texture, Time, Energy, Time-stretching, Interpolation, New Approaches to Form e infine un'appendice che riassume le trasformazioni discusse per mezzo di diagrammi. 


\section{FiELD RECORDING}

In qualsiasi composizione di un paesaggio sonoro è generalmente prevista una fase di registrazione sonora sul campo; si tratta di una pratica di ripresa audio molto particolare che, per le sue problematicità e peculiarità, necessita di determinate accortezze tecniche. Una delle pubblicazioni di riferimento sulla materia è The Handbook of Field Recording (Dorritie 2003), un manuale molto esaustivo che si prefigge lo scopo di insegnare a ottenere la miglior registrazione possibile in ogni situazione ambientale. Accompagnato da un supporto cd che contiene degli utili esempi sonori, questo testo tratta di molti argomenti di sicuro interesse, come l'audio per i documentari, le registrazioni subacquee o il sonoro dei film, oltre che approfondire le principali tematiche come l'influenza dei fattori ambientali, l'acustica degli spazi o le problematiche relative all'equipaggiamento tecnologico.

Di simile impostazione, all'apparenza, è un altro testo di riferimento, Field Recording: from Research to Wrap: an Introduction to Gathering Sound Effects (Virostek 2012), ma sebbene si occupi dei medesimi argomenti, quest'opera introduce il lettore alla figura del creative recordist e focalizza l'attenzione sulla registrazione degli effetti sonori e sulle diverse strategie di ricostruzione di determinati ambienti acustici. Poiché l'autore è uno dei massimi esperti di effetti sonori per film, la trattazione della materia privilegia la registrazione cinematografica, ma non per questo risulta meno utile o interessante anche per coloro che hanno intenzioni più dichiaratamente artistiche.

Per coloro che invece optano per una prospettiva sull'estetica della disciplina in oggetto, si consiglia la lettura di In The Field: The Art of Field Recording (Lane and Carlyle 2013). Si tratta di un'interessante raccolta di interviste ad alcuni tra i maggiori field recordist del mondo che, discutendo su una serie di opinioni e punti di vista (o per meglio dire punti di ascolto), sono in grado di aprire al lettore nuove prospettive grazie a inedite astrazioni o inusuali definizioni sulla disciplina ${ }^{11}$.

Tra i temi peculiari delle domande vi sono il processo dell'ascolto, l'oggettività della registrazione e le implicazioni concettuali della qualità di dettaglio delle registrazioni; tra la schiera degli artisti intervistati, diciotto in tutto, spiccano nomi come Hildegard Westerkamp, Steve Feld, Hiroki Sasajima, Annea Lockwood e Carsten Nicolai.

Infine, con una trattazione meno specifica dell'ambito compositivo

${ }^{11}$ Termini come audio readymade, aural photography o naturally generative artifacts. 
elettroacustico, che si allarga al suono in relazione alla letteratura, segnalo Writing the Field Recording: Sound, Word, Environment (Benson and Montgomery 2018). Il testo include 11 saggi ad opera di artisti, poeti, scrittori - tra gli autori vi sono Lisa Robertson, Carol Watts, Jonathan Skinner e John Berger - ed è corredato da registrazioni sul campo accessibili dal sito dell'editore.

\section{RIFERIMENTI BIBLIOGRAFICI}

Alcorn, P. M. 1997. Perspectives of Electroacoustic Music: a critical study of the electroacoustic music of Jonathan Harvey, Denis Smalley and Trevor Wishart. University of Durham.

Attali, J. 1978. Rumori. Saggio sull'economia politica della musica. Milano: Edizioni Gabriele Mazzotta [trad. it. Sergio Mancini].

Augoyard, J.F. and H. Torgue 2004. Repertorio degli effetti sonori. Lucca: Ricordi-LIM [trad. it. Adolfo Conrado].

Bandt, R., ed. 2007. Hearing places: sound, place, time and culture. Newcastle upon Tyne: Cambridge Scholars Publishing.

Barbosa, A. 2008. Displaced Soundscapes. Saarbrücken: VDM Verlag.

Belgiojoso, R. 2009. Costruire con i suoni. Milano: Franco Angeli.

Benson, S. and W. Montgomery, eds. 2018. Writing the Field Recording: Sound, Word, Environment. Edinburg: Edinburgh University Press.

Bergamo, F. 2018. Il disegno del paesaggio sonoro. Milano: Mimesis.

Blesser, B. and L. R. Salter. 2007. Spaces Speak, Are You Listening?. Cambridge: MIT.

Bull, M. and L. Back, eds. 2008. Paesaggi sonori. Musica, voci, rumori: l'universo dell'ascolto. Milano: Il Saggiatore.

Calanchi, A., ed. 2015. Il suono percepito, il suono raccontato. Paesaggi sonori in prospettiva. Teramo: Galaad Edizioni.

Carlson, A. and A. Berleant, eds. 2004. The Aesthetics of the Natural Environment. Peterborough: Broadview Press.

Chion, M. 1982. La musique électroacoustique. Paris: Presses Universitaires de France.

Chion, M. 1983. Guide des objets sonores: Pierre Schaeffer et la recherche musicale. Paris: Buchet/Chastel.

Chion, M. 1991. L'art des sons fixés: ou la musique concrètement. Fontaine: Metamkine/ Nota Bene/Sono Concept.

Clarke, E. 2008. Ways of Listening: An Ecological Approach to the Perception of Musical Meaning. Oxford: Oxford University Press.

Colimberti, A., a cura di. 2004. Ecologia della musica. Roma: Donzelli Editore. 
Cox, T. 2015. Sonic Wonderland: a scientific odissey of sound. London: Vintage.

Demers, J. 2010. Listening through the Noise: The Aesthetics of Experimental Electronic Music. New York: Oxford University Press.

Dorritie, F. 2003. The Handbook of Field Recording, New York: Pro Audio Press.

Emmerson, S. 1986. The Language of Electroacoustic Music. Hampshire: Palgrave Macmillan.

Erlmann, V., ed. 2008. Hearing Cultures. Essays on Sound, Listening and Modernity. Sensory formations series. Oxford: Berg.

Feld, S. 1990. Sound and Sentiment: Birds, Weeping, Poetics, and Song in Kaluli Expression. Pennsylvania: University of Pennsylvania.

Fronzi, G. 2013. Electrosound. Storia ed estetica della musica elettroacustica. Torino: EDT.

Kahn, D. 1999. Noise, water, meat: a bistory of sound in the arts. Cambridge: MIT Press.

Labelle, B. 2006. Background Noise: Perspectives on Sound Art. London: Bloomsbury Academic.

Labelle, B. 2019. Acoustic Territories: sound culture and everyday life. London: Bloomsbury Academic.

Landy, Leigh. 2007. Understanding the Art of Sound Organization. London: MIT Press. Landy, L. 2012. Making Music with Sounds. Oxon: Routledge.

Lane, C. and A. Carlyle, eds. 2013. In The Field: The Art of Field Recording. Devon: Uniformbooks.

Mayr, A., ed. 2001. Musica e suoni dell'ambiente. Bologna: CLUEB.

Nancy, J.L. 2004. All'ascolto. Milano: Raffaello Cortina Editore [tr. it. E. Lisciani Petrini].

Norman, K. 1998. Poetry of Reality. Abingdon: Taylor \& Francis.

Oliveros, P. 1984. Software for people: collected writings 1963-80. Music Series. Baltimore: Smith Publications.

Oliveros, P. 2005. Deep Listening: A Composer's Sound Practice. New York: iUniverse.

Oliveros, P. 2009. "From Outside the Window: Electronic Sound Perfomance". The Oxford Handbook of Computer Music: 467-472. New York: OUP.

Orbach, J. 1999. Sound and Music: for the pleasure of the brain. Lanham: University Press of America.

Pisano, L. 2017. Nuove geografie del suono. Spazi e territori nell'era postdigitale. Milano: Meltemi.

Radicchi, A. 2013. Sull'immagine sonora della città. Firenze: FUP.

Rothenberg, D. and M. Ulvaeus. 2009. The book of Music and Nature: an anthology of Sounds, Words, Thoughts. Middletown: Wesleyan University Press.

Russolo, L. 1916. L'arte dei rumori. Milano: Edizioni futuriste di poesia.

Schaeffer, P. 1952. A la recherche d'une musique concrète. Paris: Éditions du Seuil.

Schaeffer, P. 1966. Solfège des objets musicaux. Paris: Éditions du Seuil.

Schaeffer, P. 1977. Traité des objets musicaux. Second edition. Paris: Éditions du Seuil. 
Schaeffer, P. 2010. Essai sur la radio et le cinéma. Paris: Éditions Allia.

Schafer, M.R. 1965. The composer in the classroom. Bridgewater: BMI Canada Limited.

Schafer, M.R. 1969. The new soundscape: a bandbook for the modern music teacher. Toronto: Berandol Music

Schafer, M.R. 1970. The book of noise. Ontario: Arcana Editions.

Schafer, M.R. 1973. The Music of the Environment. Vienna: Universal Edition.

Schafer, M.R. 1977. The Soundscape: Our Sonic Environment and the Tuning of the World. New York: Alfred Knopf.

Schafer, M.R. 1985. Il paesaggio sonoro. Lucca: Ricordi-LIM [trad. it. Nemesio Ala].

Schafer, M.R. 1988. La nuova orchestra: l'universo sonoro. Milano: Suvini Zerboni [trad. it. Claudio Tommasoni].

Southworth, M. 1967. The Sonic Environment of Cities. Thesis diss., Massachussets Institute of Technology.

Toop, D. 1995. Ocean of Sound: Ambient sound and radical listening in the age of communication. London: Serpent's Tail.

Turri, E. 2004. Il paesaggio e il silenzio. Venezia: Marsilio.

Turri, E. 2006. Il paesaggio come teatro. Dal territorio vissuto al territorio rappresentato. Venezia: Marsilio.

Truax, B. 1974. "Soundscape studies: An introduction to the World Soundscape Project". Numus-West 5: 36-39.

Truax, B. 1978. Handbook for acoustic ecology. Vancouver: A.R.C. Publications

Truax, B. 2001. Acoustic Communication. Norwood, New Jersey: Ablex Pub. Corporation.

Virostek, P. 2012. Field Recording: from Research to Wrap: an Introduction to Gathering Sound Effects. Toronto: Airborne Publications.

Voegelin, S. 2014. Sonic Possible Worlds: hearing the continuum of sound. London: Bloomsbury Academic.

Weibel, P. 2019. Sound Art: sound as a medium of art. Massachussets: MIT Press.

Westerkamp, H. 1988. Listening and Soundmaking: a Study of Music as Environment. Toronto: Master of Fine Arts Thesis.

Westerkamp, H. 1999. "Soundscape Composition: Linking Inner and Outer Worlds". Organised Sound 7 (1): 51-56.

Westerkamp, H. 2002. Linking soundscape composition and acoustic ecology. Cambridge: Cambridge University Press.

Westerkamp, H. 2008. "Soundwalking”. In Autumn Leaves: Sound and the Environment in Artistic Practice, edited by Angus Carlyle, 49-54. London: University of the Arts.

Wishart, T. 1994. Audible Design: A Plain and Easy Introduction to Sound Composition. Yorkshire: Orpheus The Pantomime Ltd.

Wishart, T. 1996. On Sonic Art. Contemporary music studies 12, edited by Simon Emmerson. New York - London: Routledge. 


\section{APPENDICE: APPROFONDIMENTI}

Anderson, I. and T. Rennie. 2016. “Thoughts in the field: 'Self-reflexive narrative' in field recording." Organised Sound 21 (3): 222-232.

Alessandretti, S. and G. Sparano. 2014. "NEYMA, interactive soundscape composition based on a low budget motion capture system". Proceedings of the International Computer Music Conference 40: 379-383.

Cain, R., P. Jenning and J. Poxon. 2013. "The development and application of the emotional dimensions of a soundscape". Applied Acoustics 74 (2): 232-239.

Chapman, O. 2009. “The Icebreaker: Soundscape works as everyday sound art”. Organised Sound 14 (1): 83-88.

Chattopadhyay, B. 2012. "Sonic Menageries: Composing the sound of places". Organised Sound 17 (special issue 3): 223-229.

Davies, W.J., N. Bruce, M. Adams and M. Marselle. 2009. “The positive soundscape project: A synthesis of results from many disciplines". Internoise 38: 1-8.

Davies, W.J. 2013. "Perception of soundscapes: An interdisciplinary approach". Applied Acoustics 74 (2): 224-231.

Demers, J. 2009. "Field recording, sound art and objecthood". Organised Sound 14 (1): 39-45.

Drever, J.L. 2002. "Soundscape Composition: the convergence of ethnography and acousmatic music". Organised Sound 7 (1): 21-27.

Dumyahn, S. and Pijanowski, B. 2011. “Soundscape conservation”. Landscape Ecology 26 (9): 1327-1344.

Fells, N. 2002. "On space, listening and interaction: Words on the streets are these and Still Life". Organised Sound 7 (3): 287-294.

Foale, K. 2014. "A Listener-Centered Approach to Soundscape Analysis". PhD diss., University of Salford.

Foreman, I. 2014. "Spectral Soundscapes: Exploring Spaces of Remembrance through Sound". Interference 4.

Freeman, J., C.F. Disalvo, M. Nitsche and S. Garrett. 2011. "Soundscape Composition and Field Recording as a Platform for Collaborative Creativity". Organised Sound 16 (3): 272 - 281.

Gallagher, M. 2015. "Field recording and the sounding of spaces". Environment and Planning D - Society and Space 33 (3): 560-576.

Keller, D. 1999. Touch'n'go: Ecological Models in Composition. PhD diss., Simon Fraser University.

Keller, D. and A. Capasso. 2006. "New concepts and techniques in eco-composition". Organised Sound 11 (1): 55-62.

Koutsomichalis, M. 2013. "On soundscape, phonography and environmental sound art". Journal of Sonic Studies 4 (1). [05/02/2020]. https://www.researchcatalogue. 
net/view/268080/268081.

Ingold, T. 2007. "Against soundscape". In Autumn Leaves: Sound and the Environment in Artistic Practice, edited by Angus Carlyle, 10-13. Paris: Double Entendre.

Lane, C. 2006. "Voices from the past: compositional approaches to using recorded speech". Organised Sound 11 (1): 3-11.

López, F. 2004. "Profound Listening and Environmental Sound Matter". In Audio Culture: Readings in modern music, edited by Christoph Cox and Daniel Werner, 82-87. New York: Continuum.

Loufopoulos, A. 2005. Nature in Electroacoustic Music. PhD diss., City University London.

Loufopoulos, A. and A. Mniestries. 2011. "Soundscape models and compositional strategies in acousmatic music. Soundscape. The Journal of Acoustic Ecology 2 (1): 33-36.

Mccartney, A. 2000. "Soundscape composition and the subversion of electroacoustic norms”. Journal SEAMUS 14 (2): 6-24.

Mccartney, A. 2010. "Ethical questions about working with soundscapes”. Keynote presentation at WFAE International Conference on Ideologies and Ethics in the Uses and Abuses of Sound, Finland.

Mcgregor, I. and M. Droumeva. 2006. "Sound and Soundscape Classification: Establishing Key Auditory Dimensions and their Relative Importance”. ICAD 12: 105-112.

Meelberg, V. and M. Cobussen. 2011. "Reflections on sonic environments". Journal fo Sonic Studies 1 (1). [06/02/2020]. https://www.researchcatalogue.net/ view/166023/166040.

Misra, A., P. R. Cook, G. Wang. 2007. "Musical Tapestry: Re-composing Natural Sounds". Contemporary Music Review 36 (4): 241-250.

Parmar, R. 2012. "The Garden of Adumbrations: Reimagining environmental composition”. Organised Sound 17 (special issue 3): 202-210.

Pijanowski, B.C., L.J. Villanueva-Rivera, S.L. Dumyahn, A. Farina, B.L. Krause, B.M. Napoletano, S.H. Gage and N. Pieretti. 2011. "Soundscape ecology: the science of sound in the landscape”. BioScience 61 (3): 203-216.

Pijanowski, B.C., A. Farina, S.H. Gage, S.L. Dumyahn and B.L. Krause. 2011. "What is soundscape ecology? An introduction and overview of an emerging new science”. Landscape ecology 26 (9): 1213-1232.

Rennie, T. 2014. "Socio-Sonic: An ethnographic methodology for electroacoustic composition”. Organised Sound 19 (special issue 2): 117-124.

Rychtáriková, M. and G. Vermeir. 2013. "Soundscape categorization on the basis of objective acoustical parameters". Applied Acoustics 74 (2): 240-247.

Samuels, D.W., L. Meintjes, A.M. Ochoa and T. Porcello. 2010. "Soundscapes: Toward a sounded anthropology". Annual Review of Anthropology 39: 329-345.

Schaeffer, P. 2005. "Acoustmatics". In Audio Culture Readings in Modern Music, edited by Christoph Cox and Daniel Warner, 82-87. New York: Continuum.

Schafer, M.R. 1992. "Music, Non-Music and the Soundscape". In Companion to Con- 
temporary Musical Thought, edited by John Paynter, Tim Howell, Richard Orton and Peter Seymour, 34-45. London: Routledge.

Smalley, D. 1992. “The listening imagination: listening in the electroacoustic era". In Companion to Contemporary Musical Thought, vol. 1, edited by John Paynter, Tim Howell, Richard Orton and Peter Seymour. London: Routledge.

Truax, B. 1977. “The soundscape and technology”. Contemporary Music Review 6 (1): 1-8.

Truax, B. 1992. "Electroacoustic Music and the Soundscape: The Inner and Outer World”. In Companion to Contemporary Musical Thought, vol. 1, edited by John Paynter, Tim Howell, Richard Orton and Peter Seymour. London: Routledge.

Truax, B. 1996. "Soundscape, acoustic communication and environmental sound composition”. Contemporary Music Review 15 (1-2): 49-65.

Truax, B. 2002. "Genres and techniques of soundscape composition as developed at Simon Fraser Unibersity”. Organised Sound 7 (1): 5-13.

Truax, B. 2008. "Soundscape Composition as Global Music: Electroacoustic Music as soundscape”. Organised Sound 13 (2): 103-109.

Truax, B. 2012. "Sound, Listening and Place: The aesthetic dilemma". Organised Sound 17 (3): 193-201.

Uimonen, H. 2011. "Every Sounds Revealed: Acoustic communication and environmental recordings". Organised Sound 16 (3): 256-263.

Valle, A, V. Lombardo and M. Schirosa. 2009. "A graph-based system for the dynamic generation of soundscape". Proceedings of the International Conference on Auditory Display 15: 1-8.

Valle, A. 2010. "Simulating the soundscape through an analysis/resynthesis methodology”. Lecture Notes in Computer Science 5954: 330-357.

Westerkamp, H. 2000. “The Local and Global Language of Environmental Sound”. Proceedings of Sound Escape: An International Conference on Acoustic Ecology 1: 1-8.

Westerkamp, H. 2002. "Linking soundscape composition and acoustic ecology". Organised Sound 7 (1): 51-56.

Wrightson, K. 2000. "An introduction to acoustic ecology". Soundscape: The journal of acoustic ecology 1 (1): 10-13.

Young, J. 1996. "Imagining the Source: The Interplay of Realism and Abstraction in Electroacoustic Music". Contemporary Music Review 15 (1): 73-93. 
